\title{
Comparison of the Skin Penetration of 3 Metabolically Stable Chemicals Using Fresh and Frozen Human Skin
}

\author{
Carine Jacques-Jamin ${ }^{a}$ Hélène Duplan ${ }^{a}$ Helga Rothe $^{b}$ Ophelie Vaillant ${ }^{c}$ \\ Joan Eilstein ${ }^{d}$ Sebastien Grégoire ${ }^{d}$ Richard Cubberley ${ }^{e}$ Daniela Lange ${ }^{f}$ \\ Corie Ellison $^{9}$ Martina Klaric ${ }^{\text {h }}$ Nicola Hewitt $^{\text {h }}$ Andreas Schepky $^{f}$ \\ a Pierre Fabre Dermo-Cosmétique, Toulouse, France; ${ }^{b}$ Coty, Darmstadt, Germany; ${ }^{~}$ Eurofins I ADME Bioanalysis,

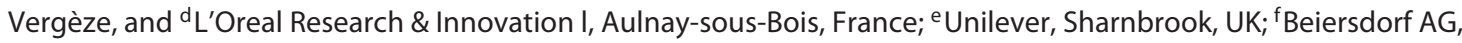

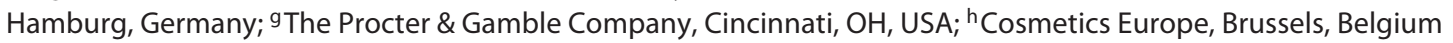

\section{Keywords}

Human skin · Fresh skin · Frozen skin · Dermal delivery ·

Penetration · Cosmetic ingredients

\begin{abstract}
Background: The Cosmetics Europe ADME Task Force is developing in vitro and in silico tools for predicting skin and systemic concentrations after topical application of cosmetic ingredients. There are conflicting reports as to whether the freezing process affects the penetration of chemicals; therefore, we evaluated whether the storage of human skin used in our studies $\left(8-12\right.$ weeks at $-20^{\circ} \mathrm{C}$ ) affected the penetration of model chemicals. Methods: Finite doses of trans-cinnamic acid (TCA), benzoic acid (BA), and 6-methylcoumarin (6MC) (non-volatile, non-protein reactive and metabolically stable in skin) were applied to fresh and thawed frozen skin from the same donors. The amounts of chemicals in different skin compartments were analysed after 24 h. Results: Although there were some statistical differences in some parameters for 1 or 2 donors, the penetration of TCA, BA, and 6MC was essentially the same in fresh and frozen skin, i.e., there were no biologically relevant differences in penetration values.
\end{abstract}

\section{KARGER}

(C) 2017 S. Karger AG, Basel

E-Mail karger@karger.com

www.karger.com/spp
Statistical differences that were evident indicated that penetration was marginally lower in frozen than in fresh skin, indicating that the barrier function of the skin was not lost. Conclusion: The penetration of the 3 chemicals was essentially unaffected by freezing the skin at $-20^{\circ} \mathrm{C}$ for up to 12 weeks.

\footnotetext{
(c) 2017 S. Karger AG, Basel
}

\section{Introduction}

The Cosmetics Europe ADME Task Force is evaluating in vitro and in silico models that can be used to determine the local and systemic bioavailability of topically applied chemicals. Measured values for critical skin penetration parameters, such as diffusion and partition coefficients, will be used to improve current in silico models for predicting the extent of penetration of chemicals across all layers of human skin. In order to assess the suc-

This collaborative work was conducted under the direction of the Cosmetics Europe Skin Bioavailability and Metabolism Task Force (now the "ADME Task Force").
Andreas Schepky

Beiersdorf AG

Unnastrasse 48

DE-20253 Hamburg (Germany)

E-Mail Andreas.Schepky@ Beiersdorf.com 
cess of the prediction and to potentially refine toxicological end point predictions, penetration of chemicals into and through ex vivo human skin will also be measured. The aim is to measure different parameters for 50 chemicals related to cosmetics ingredients, including relevant positive and negative control chemicals used to test them in genotoxicity and sensitization assays, to develop new prediction tools mimicking in vivo situations.

Established methods for determining skin penetration have been used for many decades, and there are several guidelines on how to perform skin penetration studies, including the OECD (Organization for Economic CoOperation and Development) test guidelines 28 and 428 $[1,2]$ and the SCCS (Scientific Committee on Consumer Safety) opinion [3]. The use of pig skin (as a waste product of the meat industry) for skin absorption is accepted as a surrogate for human skin in the OECD No. 28 and 428 test guidelines [1,2] and the SCCS opinion [3], although use of human skin would always be preferable. However, the supply and quality of fresh human tissue can be sporadic, and the skin pieces obtained are often too small for large experiments. Therefore, in order to be able to schedule and measure the penetration of all 50 chemicals within our target timeline of 2 years, the most practical solution would be to use frozen human or pig skin. The use of frozen skin is accepted by the OECD guidelines and SCCS opinion because skin penetration is based on passive diffusion.

Since penetration is a passive diffusion process, the penetration of metabolically stable chemicals through fresh and frozen skin samples should be similar. However, there are conflicting reports as to whether the freezing process affects the penetration of chemicals. The outcomes of the comparisons appear to depend on the physicochemical properties of chemicals tested [4-7], the skin tissues used (which vary between full-thickness [8], dermatomed $[9,10]$ and isolated epidermis layers, i.e., $100 \mu \mathrm{m}$ thick $[11,12])$, the length of storage (30-60 days [13] vs. a few hours [9]), and the parameters by which they were compared, e.g., distribution across skin layers, penetration coefficients and cumulative amounts in the receptor fluid (RF). Considering the varied reports of comparisons between fresh and frozen skin, before embarking on the main testing of 50 chemicals, we first evaluated whether the storage of human skin affected the penetration of model chemicals.

Reports in which comparisons between fresh and frozen skin were made used a variety of storage temperatures but most used approximately $-20^{\circ} \mathrm{C}$. Storage at lower temperatures (e.g., -60 or $-80^{\circ} \mathrm{C}$ ) compromises the skin
Table 1. Physicochemical properties of TCA, BA, and 6MC

\begin{tabular}{|c|c|c|c|}
\hline Parameter & TCA & $\mathrm{BA}$ & $6 \mathrm{MC}$ \\
\hline CAS & $140-10-3$ & $65-85-0$ & $92-48-8$ \\
\hline MW & 148.2 & 122.1 & 160.2 \\
\hline $\log \mathrm{P}$ & 2.13 & 1.87 & 2.25 \\
\hline Vapour pressure, $\mathrm{mm} \mathrm{Hg}$ & $5.05 \times 10^{-5}$ & 0.00158 & 0.00121 \\
\hline Melting point, ${ }^{\circ} \mathrm{C}$ & 133 & 122 & 77 \\
\hline Solubility, mg/mL & 0.79 & 4.45 & 0.49 \\
\hline Reactivity with proteins (DPRA) & none & none & none \\
\hline
\end{tabular}

$\log \mathrm{P}$ was calculated using ACD/Labs_version 12.0 [21] software for 6MC and was measured for BA [22] and TCA [23]. Melting point and vapour pressure values were all estimated using EPISuite_version 4.1 software [24]. In silico estimates were carried out using the CAS (Chemical Abstracts Service) numbers and SMILES (simplified molecular input line entry system) as references. MW, molecular weight; DPRA, direct peptide reactivity assay.

$[14,15]$. Therefore, we used a standardized storage method recommended by the OECD TG 28 guideline, such that the subcutaneous fat was removed (which makes subsequent handling easier) before wrapping the skin in aluminium foil and placing it in a $-20^{\circ} \mathrm{C}$ freezer for $8-12$ weeks. The skin was not dermatomed until after storage and subsequent thawing. For comparisons between the absorption of fresh and frozen skin from the same donors, we selected 3 chemicals that were non-volatile (based on their vapour pressure), non-protein reactive (assessed in additional experiments using the direct peptide reactivity assay, data not shown) and metabolically stable in skin (assessed in additional experiments using skin S9 subcellular fraction incubations, data not shown). These were trans-cinnamic acid (TCA), benzoic acid (BA), and 6-methylcoumarin (6MC), all of which are used as cosmetic ingredients. The physicochemical properties of the chemicals are summarized in Table 1. These also represented chemicals with low (TCA), moderate $(\mathrm{BA})$, and high $(6 \mathrm{MC})$ skin penetration properties.

\section{Materials and Methods}

\section{Chemicals}

TCA [ring- ${ }^{14} \mathrm{C}(\mathrm{U})$ ] (98\% pure; specific activity: $2,035 \mathrm{MBq} /$ $\mathrm{mmol}$ ) and BA [ring- $\left.{ }^{14} \mathrm{C}(\mathrm{U})\right]$ (99.9\% pure; specific activity: 4,810 $\mathrm{MBq} / \mathrm{mmol}$ ) were from ARC Inc., St. Louis, MO, USA. 6-Methyl $\left[2-{ }^{14} \mathrm{C}\right]$ coumarin $(99.9 \%$ pure; specific activity: $380 \mathrm{MBq} / \mathrm{mmol}$ ) was from Selcia, Ongar, UK. The label-free chemicals TCA, BA, 
and 6MC were from Sigma-Aldrich (St. Louis, MO, USA). All other chemicals and solutions used were from Sigma-Aldrich.

Radiolabelled chemicals, as tracers, were mixed with label-free chemicals to achieve the final concentrations (in \% w/v) and the same isotopic concentrations $(1,110 \mathrm{~Bq} / \mu \mathrm{L})$. The solvent for all 3 chemicals was phosphate-buffered saline (PBS) with a phosphate concentration of $0.1 \mathrm{M}, \mathrm{pH} 7.4$. The final concentrations (w/v) of BA, TCA, and $6 \mathrm{MC}$ were $0.075 \%(0.75 \mathrm{mg} / \mathrm{mL}), 0.4 \%(4 \mathrm{mg} / \mathrm{mL})$, and $0.047 \%(0.47 \mathrm{mg} / \mathrm{mL})$, respectively. The concentrations were based on the maximal solubility in water and, although these were different, they represented finite doses. The homogeneity of the dosing solution was controlled with 6 aliquots (even distribution on the upper, middle, and lower layers of the formulation). The homogeneity, expressed as the percent coefficient of variation of the distribution in the formulation layers, was $1.2-3.3 \%$ for TCA, $0.9-3.4 \%$ for $\mathrm{BA}$, and $1.6-7.0 \%$ for $6 \mathrm{MC}$. Following the application of $10 \mu \mathrm{L} / \mathrm{cm}^{2}$ to $1 \mathrm{~cm}^{2}$ of skin, the total applied ${ }^{14} \mathrm{C}$ radioactivity was $11,100 \mathrm{~Bq}$. The radiopurity of all 3 formulations was greater than $98 \%$ before application to the skin and after $24 \mathrm{~h}$ incubation at $32^{\circ} \mathrm{C}$ (measured by radio-HPLC).

\section{Skin Tissue}

Abdominal human skin was ethically obtained with consent for research from donors undergoing surgery from commercial suppliers and in accordance with French laws (Banque de Tissu Lyon, France, Biopredic International, Saint-Grégoire, France, and Alphenyx, Marseille, France). The skin was devoid of obvious damage, disease, or stretch marks. The donors used for comparing fresh and frozen skin were all female Caucasians of different ages: donor 1: 37 years; donor 2: 52 years; donor 3: 45 years; donor 4: 53 years; donor 5: 39 years; donor 6: 34 years; donor 7: 52 years. The donors used for comparing the effect of RF collection tubing were also all female Caucasians with ages between 36 and 57. Excess subcutaneous fat was removed before wrapping the skin in wetted (with PBS) aluminium foil and shipping at $4^{\circ} \mathrm{C}$ overnight. The fresh skin was either used fresh or frozen within $24 \mathrm{~h}$ of shipment. Part of the skin sample was used in "fresh" viable skin studies, and the remaining part was placed in aluminium foil and stored at $-20^{\circ} \mathrm{C}$ for $8-12$ weeks. The skin discs were thawed at room temperature for 30-45 min and dabbed dry using cellulose swabs. For each chemical, 3 replicate discs from each of 4 donors were used, with the exception of donor 6 for $6 \mathrm{MC}$, which used 2 discs. Human skin was dermatomed (using an electric dermatome) to a thickness of $400 \pm 50 \mu \mathrm{m}$, which was measured using Oditest callipers.

\section{Skin Penetration Experiment}

Flow-through cells with a $1-\mathrm{cm}^{2}$ application area were used. The receptor compartment of the diffusion cell was filled with RF $(0.9 \% \mathrm{NaCl}$ in water, supplemented with $1 \%(\mathrm{w} / \mathrm{v})$ bovine serum albumin and $0.05 \%(\mathrm{v} / \mathrm{v})$ gentamycin sulphate). Skin discs were placed onto the receptor compartment of a diffusion cell, and the donor compartment was then placed onto the skin, and both compartments were tightly clamped. All experiments were conducted using non-occluded conditions. The temperature of the diffusion chamber and skin was measured at the start of the experiment and maintained throughout the assay at $32 \pm 2{ }^{\circ} \mathrm{C}$. The flow rate of the $\mathrm{RF}$ was $1 \mathrm{~mL} / \mathrm{h}$. Once mounted, the diffusion cells were allowed to equilibrate for $1 \mathrm{~h}$. After equilibration, the skin integrity was confirmed before application of chemicals by measuring the transepidermal water loss (TEWL) using a Tewameter. Only skin discs with a TEWL between 0.7 and $5 \mathrm{~g} \times \mathrm{m}^{-2} \times \mathrm{h}^{-1}$ were used in the experiments.

A volume of $10 \mu \mathrm{L} / \mathrm{cm}^{2}$ of PBS buffer containing each chemical was applied to the surface of the skin $\left(1 \mathrm{~cm}^{2}\right)$ using a positive displacement pipette and spread (without pressure) to ensure an even distribution of the solution. The RF was sampled $1 \mathrm{~h}$ prior to dosing and then after dosing at $30 \mathrm{~min}, 1,2,4,8,16$, and $24 \mathrm{~h}$. The stability of the chemical in the formulation over $24 \mathrm{~h}$ at $32^{\circ} \mathrm{C}$ was tested by radio-HPLC, confirming the presence of a single peak co-eluting with the retention time of the parent chemical.

After $24 \mathrm{~h}$, the skin surface was washed with $0.5 \mathrm{~mL}$ water containing $10 \%$ Tween $80{ }^{\circledR}$ using a cotton bud and gentle rubbing for $30 \mathrm{~s}$. The skin was then washed 7 times with $0.5 \mathrm{~mL}$ water and then carefully dried using cotton buds. The skin wash solutions, cotton buds and tips were collected for analysis. After the skin wash, the cell system was dismantled. The donor and receptor compartments were placed in flasks and $10 \mathrm{~mL}$ and $40 \mathrm{~mL}$ ethanol was added, respectively, and the closed flasks were shaken at least overnight. The skin was tape stripped using adhesive scotch tape Magic $3 \mathrm{M}$, with a weight of $150 \mathrm{~g} / \mathrm{cm}^{2}$ placed on top of the Scotch tape for $10 \mathrm{~s}$ before removal. A maximum of 20 strips were taken and pooled in a vial as follows: strips $1-2,3-8,9-14$, and 15-20. Soluene ${ }^{\circledR}-350(3 \mathrm{~mL})$ was added, and the vial was placed at approximately $60^{\circ} \mathrm{C}$ until the stratum corneum (SC) samples were dissolved. The epidermis and partial dermis were separated by heating at $60^{\circ} \mathrm{C}$ for $40 \mathrm{~s}$ and using a scalpel blade. Soluene ${ }^{\circledR}-350(3 \mathrm{~mL})$ was added, and the vials were heated at approximately $60^{\circ} \mathrm{C}$ until the samples were dissolved. Appropriate volumes of Ultima Gold scintillation liquid were added to each of the samples, which were then analysed for radioactivity using a scintillation counter.

\section{Calculations and Statistics}

The dermal absorption was calculated as the total amount present within the epidermis (without SC), dermis and RF. The mean of replicates for each donor and that of all replicates/donors were compared using the 2-tailed paired Student test, using Instat (Graph-Pad software), where a statistical significance was reached if $p<0.05$.

\section{Results}

\section{Skin Thickness and TEWL}

The mean thicknesses of fresh and frozen skin discs used in these studies were comparable and were $423 \pm 23$ $\mu \mathrm{m}$ (range 358-446 $\mu \mathrm{m}$ ) and $409 \pm 27 \mu \mathrm{m}$ (range 356-448 $\mu \mathrm{m})$, respectively. The skin integrity test using TEWL demonstrated that the mean frozen value $(1.85 \pm 0.99 \mathrm{~g} \times$ $\left.\mathrm{m}^{-2} \times \mathrm{h}^{-1}\right)$, although more variable, was not significantly different ( $t$ test, $p>0.05)$, from the fresh value (1.51 \pm

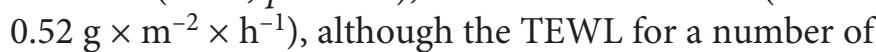
skin discs used for TCA were higher in frozen than in fresh skin from the same donor (e.g., donor 1, disc 1: 1.28 and $2.96 \mathrm{~g} \times \mathrm{m}^{-2} \times \mathrm{h}^{-1}$ in fresh and frozen skin, respectively) (Fig. 1). Equally, there were some TEWL values that were lower than the corresponding value in paired donor skin. The TEWL values did not correlate with the 
Table 2. Amount of TCA, BA, and $6 \mathrm{MC}$ entering the RF $24 \mathrm{~h}$ after application to fresh and frozen skin

\begin{tabular}{|c|c|c|c|c|c|}
\hline \multirow[t]{2}{*}{ Chemical } & \multirow[t]{2}{*}{ Donor } & \multicolumn{2}{|c|}{ Amount entering RF, \% applied dose } & \multicolumn{2}{|c|}{ Amount entering RF, $\mu \mathrm{g} / \mathrm{cm}^{2}$} \\
\hline & & fresh & frozen & fresh & frozen \\
\hline \multirow[t]{5}{*}{ TCA } & Donor 1 & $6.5 \pm 2.8$ & $11.0 \pm 7.6$ & $0.52 \pm 0.23$ & $0.86 \pm 0.61$ \\
\hline & Donor 2 & $24.7 \pm 2.2$ & $13.8 \pm 4.5^{*}$ & $1.95 \pm 0.18$ & $1.03 \pm 0.33^{*}$ \\
\hline & Donor 3 & $8.4 \pm 1.2$ & $9.7 \pm 5.7$ & $0.68 \pm 0.10$ & $0.75 \pm 0.43$ \\
\hline & Donor 4 & $12.7 \pm 4.1$ & $7.4 \pm 0.8$ & $1.03 \pm 0.34$ & $0.68 \pm 0.16$ \\
\hline & All & $13.8 \pm 7.7$ & $10.5 \pm 5.1$ & $1.04 \pm 0.61$ & $0.83 \pm 0.38$ \\
\hline \multirow[t]{5}{*}{ BA } & Donor 2 & $45.7 \pm 6.0$ & $22.2 \pm 5.3^{*}$ & $19.2 \pm 2.5$ & $9.1 \pm 2.3^{*}$ \\
\hline & Donor 3 & $32.0 \pm 4.7$ & $33.7 \pm 7.0$ & $13.5 \pm 2.2$ & $13.9 \pm 2.6$ \\
\hline & Donor 5 & $24.1 \pm 6.3$ & $13.8 \pm 6.9$ & $10.2 \pm 2.8$ & $5.6 \pm 2.8$ \\
\hline & Donor 6 & $45.8 \pm 8.7$ & $47.6 \pm 18.4$ & $19.2 \pm 3.9$ & $19.4 \pm 7.2$ \\
\hline & All & $36.9 \pm 11.2$ & $29.4 \pm 16.1$ & $15.5 \pm 4.7$ & $12.0 \pm 6.5$ \\
\hline \multirow[t]{5}{*}{$6 \mathrm{MC}$} & Donor 1 & $71.6 \pm 3.3$ & $78.1 \pm 4.0$ & $3.5 \pm 0.1$ & $3.7 \pm 0.1$ \\
\hline & Donor 5 & $80.8 \pm 4.8$ & $63.3 \pm 1.3^{*}$ & $3.9 \pm 0.2$ & $3.1 \pm 0.1^{*}$ \\
\hline & Donor 6 & $82.5 \pm 0.9$ & $74.5 \pm 5.0$ & $4.0 \pm 0.0$ & $3.6 \pm 0.2$ \\
\hline & Donor 7 & $72.7 \pm 3.0$ & $57.8 \pm 5.9$ & $3.5 \pm 0.1$ & $2.9 \pm 0.3$ \\
\hline & All & $76.4 \pm 5.7$ & $68.0 \pm 8.9$ & $3.7 \pm 0.2$ & $3.3 \pm 0.4$ \\
\hline
\end{tabular}

Values are means \pm SD. Statistically different $(p<0.05)$ values are denoted with an asterisk.

dermal delivery of the chemical (data not shown) or the amount reaching the RF (Table 2) since the values were all well below the upper limit for this parameter $(5 \mathrm{~g} \times$ $\left.\mathrm{m}^{-2} \times \mathrm{h}^{-1}\right)$.

\section{Cumulative Absorption}

The cumulative absorption of each chemical into the $\mathrm{RF}$ showed the tendency to be slightly lower for frozen skin than fresh skin but the difference in mean values for all 4 donors was not statistically significant for any of the time points (Fig. 2). For individual donors, the only statistically significant $(p<0.05)$ differences in the total amount entering the RF were for TCA in donor $2(24.7 \%$ in fresh compared to $13.8 \%$ in frozen skin), BA in donor 2 (45.7\% in fresh compared to $22.2 \%$ in frozen skin), and $6 \mathrm{MC}$ in donor 5 (80.8\% in fresh compared to $63.38 \%$ in frozen skin) (Table 2). As with the cumulative absorption, only few data from individual donors showed statistically significant differences in the total amount entering the RF.

\section{Distribution across Skin Layers}

Figure 3 shows the distribution of TCA in the RF and across the layers of fresh and frozen skin from 4 donors. The majority of the dose was washed from the surface of the skin at the end of the experiment (a mean of $83 \pm 10$

Skin Penetration in Fresh and Frozen Human Skin

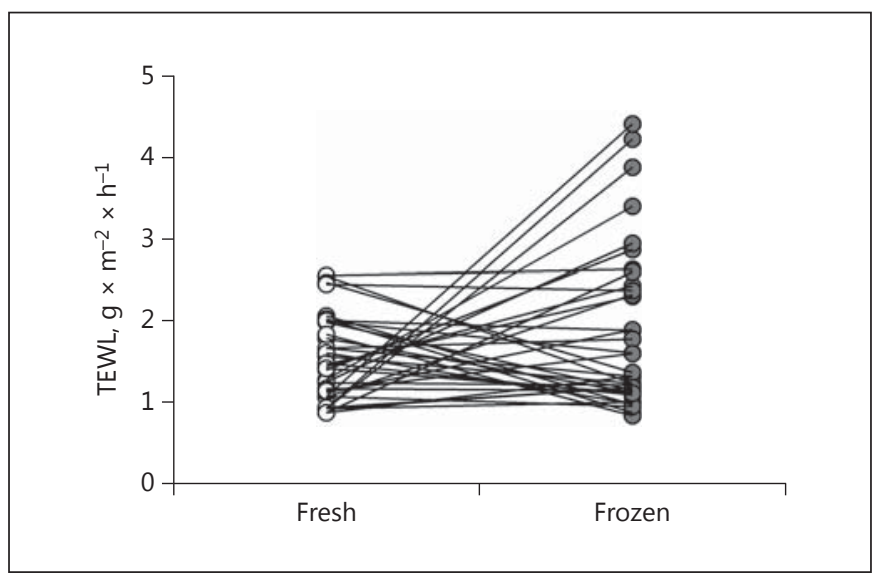

Fig. 1. TEWL values for fresh and frozen skin used for all 3 chemicals. The lines between data points link fresh $(O)$ and frozen ( skin from the same donor.

and $89 \pm 7 \%$ of the applied dose in fresh and frozen skin from all 4 donors, respectively). Very little of the dose was detected in the lower skin layers and SC of fresh and frozen skin $(<1.2 \%$ of the applied dose). The amount of TCA entering the RF (a mean of $11 \%$ of the applied dose in fresh and frozen skin from all 4 donors) was much lower than for BA (Fig. 4) and 6MC (Fig. 5), and there was no 

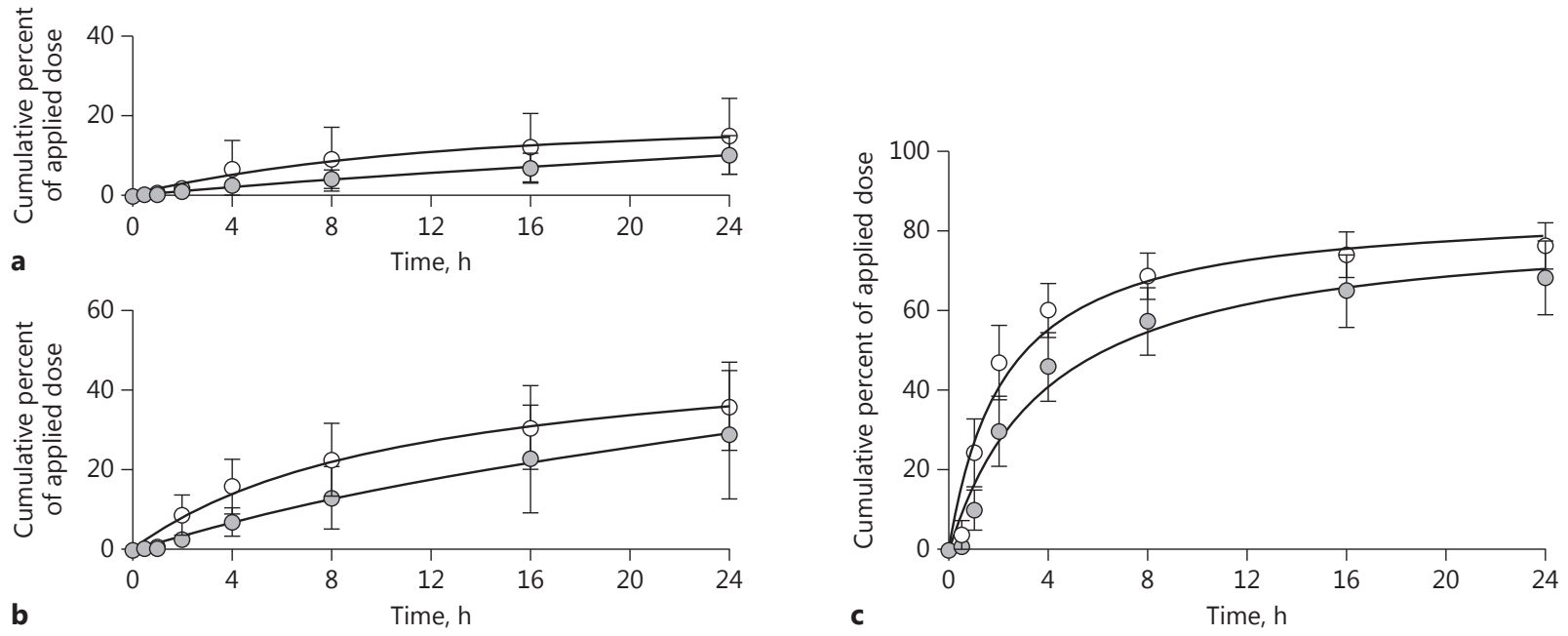

Fig. 2. Cumulative amounts of TCA (a), BA (b), and $6 \mathrm{MC}(\mathbf{c})$ in the RF after topical application to fresh $(\mathrm{O})$ and frozen $(O)$ human skin. Values are expressed as the cumulative percentage of the applied dose and are a mean \pm $\mathrm{SD}$ of 12 skin discs (triplicate discs from 4 donors, with the exception of donor 3 for $6 \mathrm{MC}$ which was in duplicate).

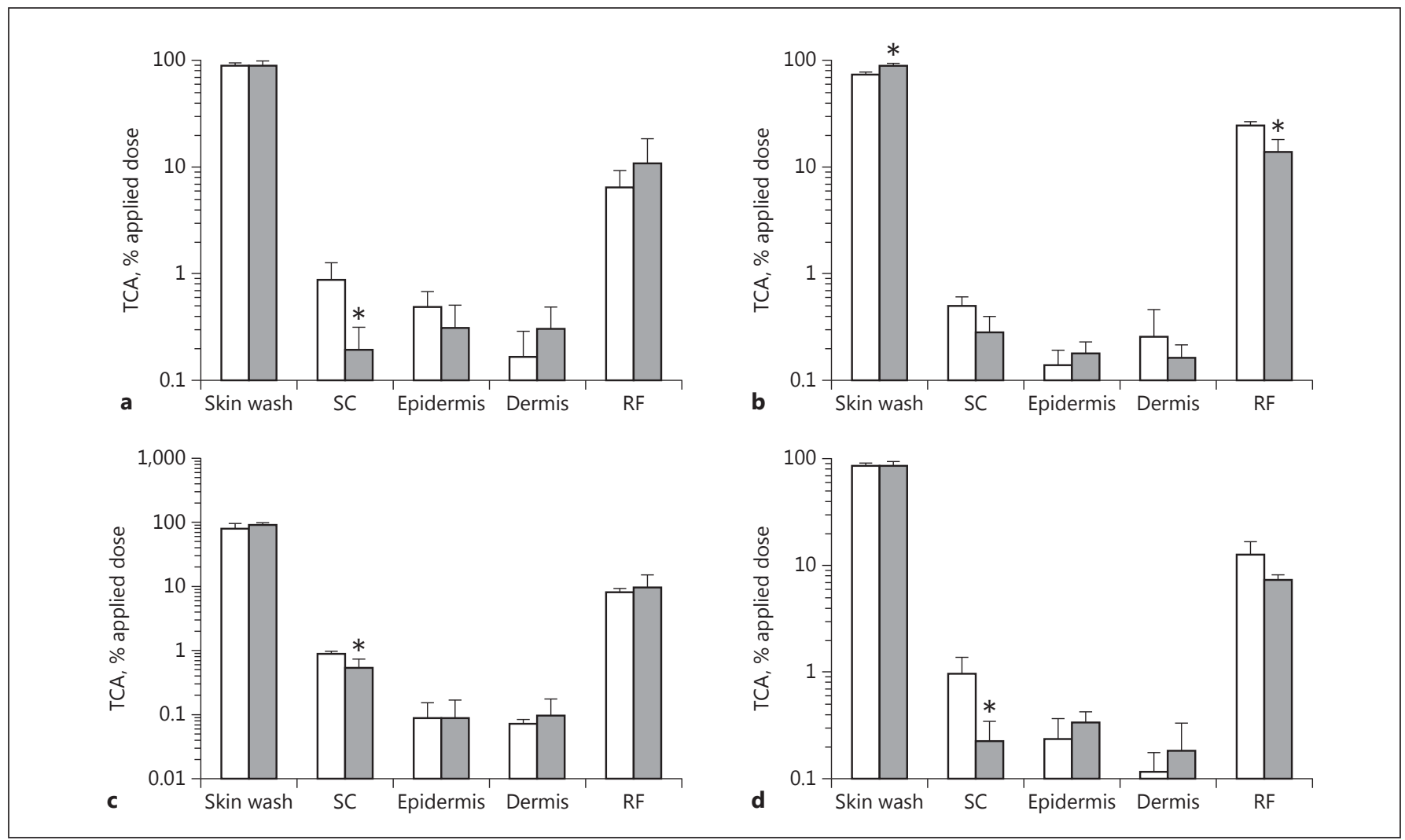

Fig. 3. Distribution of radiolabelled TCA across different layers of human skin, skin wash, and RF $24 \mathrm{~h}$ after topical application to fresh $(\square)$ and frozen $(\square)$ skin. Values are a mean \pm SD of 3 skin discs and 4 donors (12 in total). Statistical differences $(p<0.05)$ between fresh and frozen values are denoted with an asterisk. a Donor 1. b Donor 2. c Donor 3. d Donor 4 . 


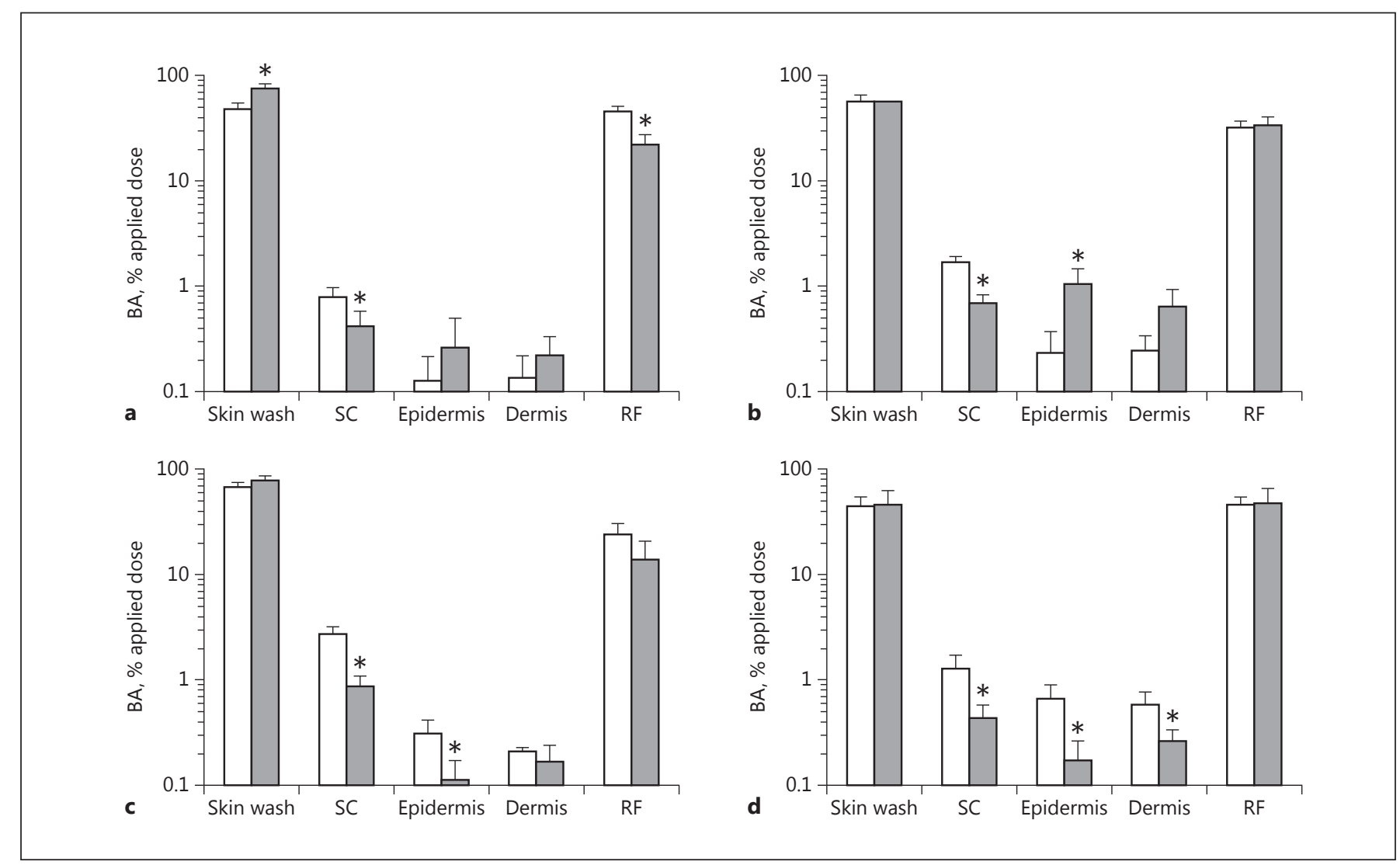

Fig. 4. Distribution of radiolabelled BA across different layers of human skin, skin wash, and RF $24 \mathrm{~h}$ after topical application to fresh $(\square)$ and frozen $(\square)$ skin. Values are a mean \pm SD of 3 skin discs and 4 donors (12 in total). Statistical differences $(p<0.05)$ between fresh and frozen values are denoted with an asterisk. a Donor 2. b Donor 3. c Donor 5. d Donor 6.

difference between the amount detected in this compartment in fresh and frozen skin incubations, with the exception of donor 2. Here the amount of TCA in the RF was significantly higher in fresh $(24.7 \pm 2.2 \%$ of the applied dose) than in frozen $(13.8 \pm 4.5 \%$ of the applied dose) skin incubations (Fig. 3b; Table 2). Concomitant with this, there was a significantly higher amount of TCA detected in the skin wash of the frozen skin $(89.9 \pm 4.2 \%$ of the applied dose) compared to the fresh skin (74.9 \pm $3.2 \%$ ) of this donor.

Figure 4 shows the distribution of BA in the RF and across the layers of fresh and frozen skin from 4 donors. The majority of the dose was washed from the surface of the skin at the end of the experiment (a mean of $55 \pm 12$ and $64 \pm 16 \%$ of the applied dose in fresh and frozen skin from all 4 donors, respectively). Very little of the dose remained in the SC or the lower skin layers (all layers representing $<2.3 \%$ of the applied dose in fresh and frozen

Skin Penetration in Fresh and Frozen Human Skin skin). The amounts detected in the RF were in the range of $14-46 \%$ and varied between the skin donors, but were comparable within donors: donor $2=45.7 \%$ (fresh) and $22.4 \%$ (frozen); donor $3=32.0 \%$ (fresh) and $33.7 \%$ (frozen); donor $5=24.1 \%$ (fresh) and $13.8 \%$ (frozen); donor $6=45.8 \%$ (fresh) and $47.6 \%$ (frozen). Although the amount of BA in RF from donor 2 was significantly lower in frozen skin than the corresponding fresh skin (Fig. 4a; Table 2), the mean difference across all donors was not statistically different (Table 2). This was concomitant with a significantly higher amount of BA being recovered from the skin surface in frozen skin $(76 \pm 7 \%)$ compared to that from fresh skin from the same donor $(48 \pm 8 \%)(p<0.05$, Fig. $4 a)$.

Figure 5 shows the distribution of $6 \mathrm{MC}$ in the RF and across the layers of fresh and frozen skin from 4 donors. In each case, the majority of the dose was detected in the RF (a mean of $76 \pm 6$ and $68 \pm 9 \%$ of the applied dose for 


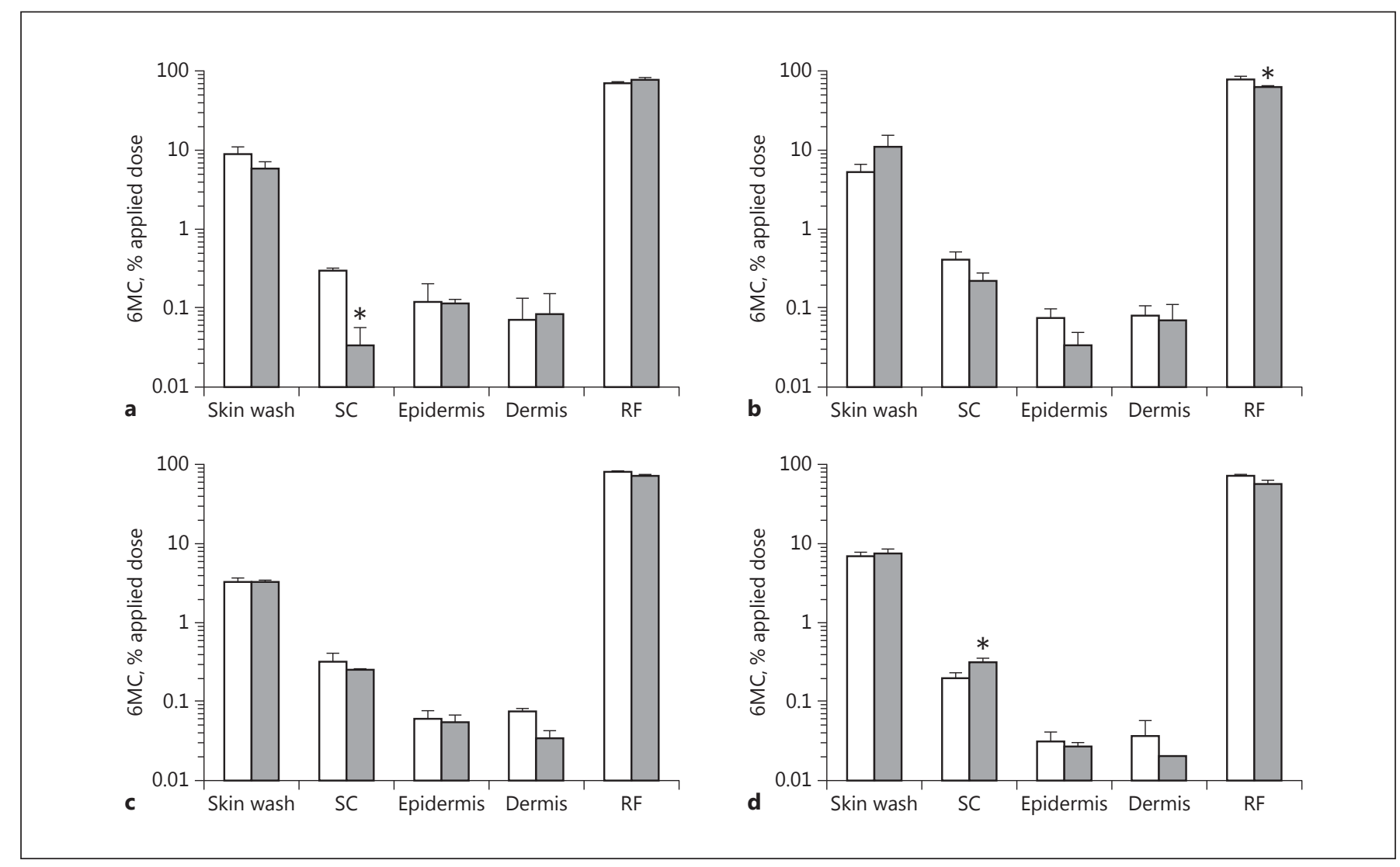

Fig. 5. Distribution of radiolabelled 6MC across different layers of human skin, skin wash, and RF $24 \mathrm{~h}$ after topical application to fresh $(\square)$ and frozen $(\square)$ human skin. Values are a mean \pm SD of 3 skin discs and 4 donors (12 in total with the exception of donor 6 for $6 \mathrm{MC}$ which was in duplicate, total of 11 discs). Statistical differences $(p<0.05)$ between fresh and frozen value are denoted with an asterisk. a Donor 1. b Donor 5. c Donor 6. d Donor 7 .

fresh and frozen skin, respectively), and very little was recovered from the skin wash $(6 \pm 2$ and $7 \pm 4 \%$ of the applied dose for fresh and frozen skin, respectively) or from the SC or viable skin layers $(<0.5 \%$ of the applied dose was present in all 3 layers of fresh and frozen skin). The amount of $6 \mathrm{MC}$ in the RF of skin incubations with donor 5 was statistically lower in the frozen skin $(63.3 \%$ of the applied dose) than in the corresponding fresh skin of the same donors ( $80.8 \%$ of the applied dose; Fig. 5 b; Table 2 ).

\section{Dermal Delivery and Mass Balance}

Table 3 shows the dermal delivery (epidermis plus dermis plus RF - the amount in the SC is also listed) of all 3 chemicals in fresh and frozen skin. Although there was a statistically significant difference in the dermal delivery of $6 \mathrm{MC}$ in donor 5 , the mean values for this chemical in all 4 donors were the same in fresh and frozen skin.
The mass balance of TCA and BA was within the 85 and $115 \%$ range recommended in the OECD and SCCS guidelines. For $6 \mathrm{MC}$, the mass balance of $6 \mathrm{MC}$ was lower than for the other 2 chemicals $(66-87 \%$ compared to $>92 \%$ for BA and $>95 \%$ for TCA). Additional investigations indicated that the low mass balance was due to the binding of $6 \mathrm{MC}$ to the PVC tubing, causing lower amounts to be recovered in the RF (Fig. 6a). Experiments using only frozen skin from 4 additional donors indicated that the mass balance of $6 \mathrm{MC}$ was increased from $64 \pm 28$ to $97 \pm 3 \%$ by using low binding fluorinated ethylene propylene (FEP)-Teflon tubing, which resulted in a higher amount recovered in the RF compared to the PVC tubing. By contrast, the amounts of $6 \mathrm{MC}$ in the skin wash and skin layers were unaffected by the type of tubing used (Fig. 6b). Using data from all 16 experiments for $6 \mathrm{MC}$ (with and without low binding tubing), the amount of 
Table 3. Dermal absorption of TCA, BA, and 6MC in fresh and frozen skin

\begin{tabular}{|c|c|c|c|c|c|}
\hline \multirow[t]{2}{*}{ Chemical } & \multirow[t]{2}{*}{ Donor } & \multicolumn{2}{|c|}{ Epidermis, dermis, and RF } & \multicolumn{2}{|l|}{ SC } \\
\hline & & fresh & frozen & fresh & frozen \\
\hline \multirow[t]{5}{*}{ TCA } & Donor 1 & $0.57 \pm 0.24$ & $0.91 \pm 0.62$ & $0.07 \pm 0.03$ & $0.01 \pm 0.01$ \\
\hline & Donor 2 & $1.98 \pm 0.17$ & $1.05 \pm 0.32$ & $0.04 \pm 0.01$ & $0.02 \pm 0.01$ \\
\hline & Donor 3 & $0.69 \pm 0.11$ & $0.76 \pm 0.44$ & $0.07 \pm 0.01$ & $0.04 \pm 0.02$ \\
\hline & Donor 4 & $1.06 \pm 0.35$ & $0.72 \pm 0.18$ & $0.08 \pm 0.03$ & $0.02 \pm 0.101$ \\
\hline & All & $1.08 \pm 0.64$ & $0.86 \pm 0.15$ & $0.06 \pm 0.03$ & $0.02 \pm 0.02$ \\
\hline \multirow[t]{5}{*}{$\mathrm{BA}$} & Donor 2 & $19.3 \pm 2.5$ & $9.3 \pm 2.4$ & $0.34 \pm 0.08$ & $0.17 \pm 0.06$ \\
\hline & Donor 3 & $13.7 \pm 2.2$ & $14.6 \pm 2.4$ & $0.72 \pm 0.08$ & $0.29 \pm 0.06$ \\
\hline & Donor 5 & $10.4 \pm 2.8$ & $5.7 \pm 2.8$ & $1.14 \pm 0.20$ & $0.35 \pm 0.08$ \\
\hline & Donor 6 & $19.7 \pm 4.0$ & $19.6 \pm 7.2$ & $0.53 \pm 0.17$ & $0.18 \pm 0.06$ \\
\hline & All & $15.8 \pm 4.8$ & $12.3 \pm 6.6$ & $0.68 \pm 0.34$ & $0.25 \pm 0.10$ \\
\hline \multirow[t]{5}{*}{$6 \mathrm{MC}$} & Donor 1 & $3.53 \pm 0.13$ & $3.71 \pm 0.12$ & $0.014 \pm 0.001$ & $0.002 \pm 0.001$ \\
\hline & Donor 5 & $3.89 \pm 0.19$ & $3.05 \pm 0.08^{\star}$ & $0.020 \pm 0.005$ & $0.010 \pm 0.003$ \\
\hline & Donor 6 & $3.96 \pm 0.02$ & $3.64 \pm 0.19$ & $0.015 \pm 0.004$ & $0.012 \pm 0.001$ \\
\hline & Donor 7 & $3.52 \pm 0.13$ & $2.85 \pm 0.29$ & $0.009 \pm 0.001$ & $0.016 \pm 0.002$ \\
\hline & All & $3.70 \pm 0.24$ & $3.31 \pm 0.42$ & $0.015 \pm 0.005$ & $0.010 \pm 0.006$ \\
\hline
\end{tabular}

The dermal delivery is defined as the sum of the amount $\left(\mu \mathrm{g} / \mathrm{cm}^{2}\right)$ in the epidermis, dermis, and RF, without the amount in the SC, which is also listed. Values are means \pm SD of 3 discs from each donor. a statistically different $(p<0.05)$ value is denoted with an asterisk.

$6 \mathrm{MC}$ in the RF could be correlated to the mass balance of the experiment (Fig. 7). The lowest mass balance and RF values were from frozen skin from donor 5, which accounts for the difference seen between fresh and frozen skin for this donor. All other experiments comparing the skin penetration between fresh and frozen skin had mass balances between 75 and $87 \%$, which resulted in more comparable dermal delivery values between fresh and frozen skin. We considered the conclusions from the experiments comparing fresh and frozen skin as valid because (a) we could account for the lower mass balance, (b) the cutaneous distribution of $6 \mathrm{MC}$ was not affected by the use of the PVC tubing, and (c) the level of binding to tubing was similar in each experiment, i.e., a good reproducibility despite this effect.

\section{Penetration across SC Layers}

The total amount of each chemical in the SC is listed in Table 3. The SC samples were pooled as follows: the first 2 strips and then the following strips 3-8, 9-14 and 15-20. The amount of chemical per strip was then calculated by dividing by 2 (pooled 1-2 strips) or by 6 (for each of the subsequent pooled samples). The mean amount of TCA per tape strip taken from the first 1-8 strips of SC of skin was lower in frozen than in fresh skin (Fig. 8a). The

Skin Penetration in Fresh and Frozen Human Skin amount of TCA per strip in lower SC layers was comparable in frozen and in fresh skin (strips 9-20, Fig. 8a). The total amount of BA per tape strip in the SC was lower when the skin was frozen, which was evident across most tape strips from this compartment (Fig. 8b). The amount of 6MC per tape strip across different layers of SC of skin from different donors was comparable in fresh and frozen skin (Fig. 8c).

\section{Discussion}

We established that the storage of skin samples for $8-12$ weeks at $-20^{\circ} \mathrm{C}$ did not change significantly the penetration (i.e., dermal delivery or the kinetics of accumulation into the RF) of 3 metabolically stable chemicals across human skin from the same donors compared to donor-matched fresh skin. Consequently, these storage conditions did not compromise the barrier function of the skin. A number of parameters were measured to reflect the overall effect of freezing, namely TEWL, distribution across skin layers, and dermal delivery $( \pm S C)$.

The conditions by which the studies were conducted were according to the harmonized protocol which will be used for a Cosmetics Europe programme and is described

Skin Pharmacol Physiol 2017;30:234-245 241 


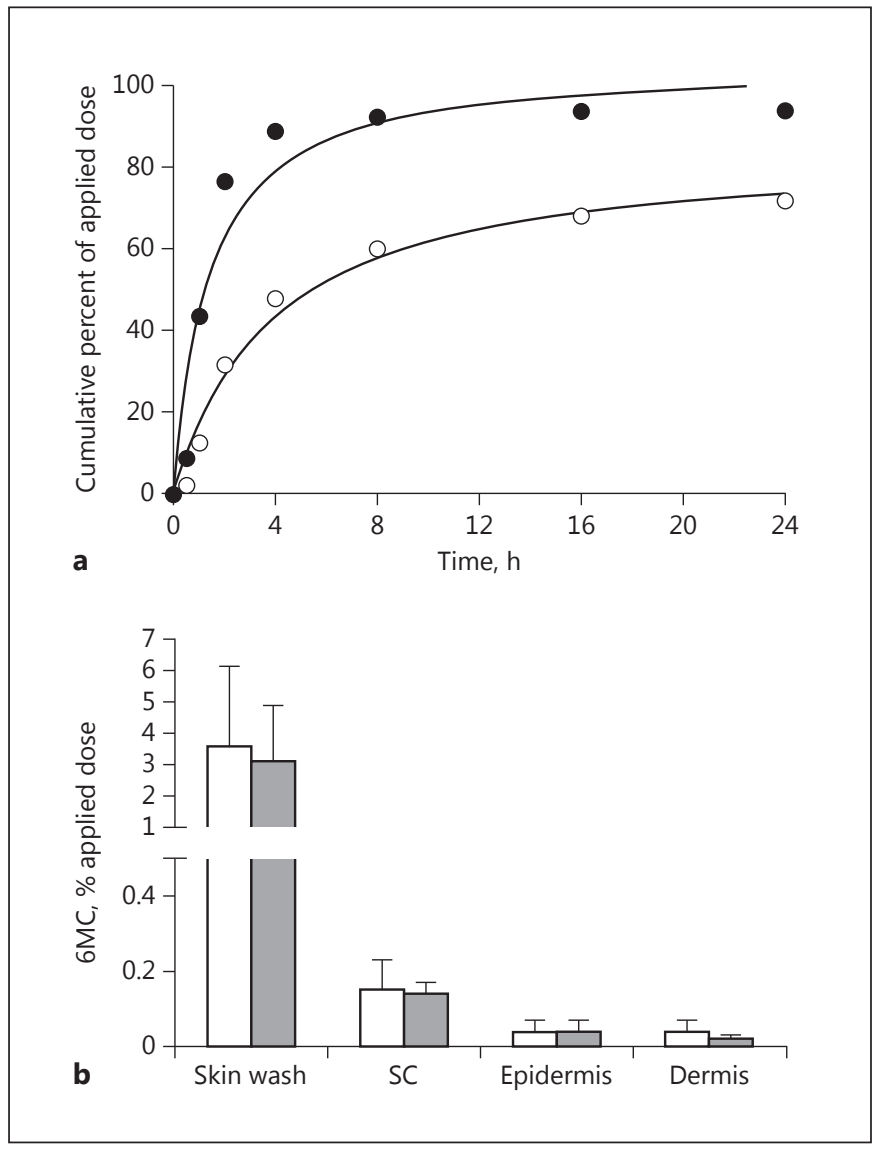

Fig. 6. Effect of tubing on the cumulative amounts of $6 \mathrm{MC}$ in the RF (a) and its cutaneous distribution (b), after topical application to frozen human skin. RF was collected using PVC tubing $(O, \square)$ and FEP-Teflon tubing $(\mathbf{O}, \mathbf{\square})$. Values are a mean \pm SD of 3 skin discs and 4 donors (12 in total - the same skin donors were used for both tubing types; with the exception of donor 6 for $6 \mathrm{MC}$ which was in duplicate, total of 11 discs).

by Gerstel et al. [16], such that fresh and frozen (stored at $-20^{\circ} \mathrm{C}$ for $8-12$ weeks) skin was dermatomed to $400 \pm$ $50 \mu \mathrm{m}$ before discs were punched and used in the assays. Results from fresh and frozen skin were compared from the same donors to avoid effects due to donor variation, and demonstrated a very good reproducibility of penetration values within and between donors, which allowed for pooling of data. In our studies, the doses of TCA, BA, and $6 \mathrm{MC}$ were finite doses based on their maximum solubility in water $\left(10 \mu \mathrm{L} / \mathrm{cm}^{2}\right.$ at $0.047,0.4$ and $0.075 \%(\mathrm{w} / \mathrm{v})$, respectively), which reflected typical in-use conditions in cosmetic products and are in accordance with the SCCS guidelines for testing.

The TEWL is a marker for the water barrier function of the skin such that the amount of water that evaporates

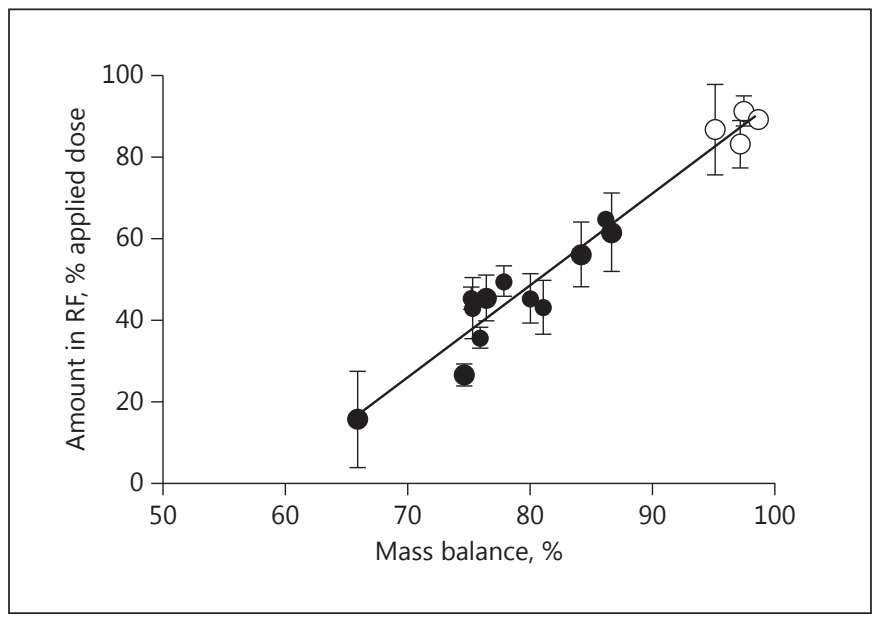

Fig. 7. Correlation between the mass balance and the amount of $6 \mathrm{MC}$ in the RF in experiments using PVC $(\mathrm{O})$ and FEP-Teflon tubing $(\bigcirc)$. Data are from 16 experiments using fresh (4 experiments) or frozen (12 experiments) skin. Values are expressed as a percentage of the applied dose and are a mean \pm SD of 3 skin discs per donor.

from the skin surface increases as the SC barrier is damaged [17]. In these studies, all the TEWL values were below the $5 \mathrm{~g} \times \mathrm{m}^{-2} \times \mathrm{h}^{-1}$ acceptance limit; moreover, freezing the skin did not necessarily result in a higher TEWL value (since TEWL values for frozen skin discs were both lower and higher than that for fresh skin from the same donor). The penetration of chemicals was also not dependent on the TEWL values measured, which is in line with the measured intact barrier function and the findings of others [8].

The frozen skin used in these studies was not dermatomed until after storage and subsequent thawing. The skin thickness may have an impact on the effect of freezing since others who compared fresh and frozen skin with the dermis removed showed significant increases in the penetration of chemicals after freezing the skin. For example, Nácher et al. [11] compared the integrity of fresh and frozen epidermal membranes using phenol red as a marker of skin integrity and acyclovir as a hydrophilic model compound. The penetration of both compounds was much higher in frozen skin (stored at $-20^{\circ} \mathrm{C}$ for 3 months). Likewise, Swarbrick et al. [9] reported higher penetration of a test chemical through human SC/epidermal layers (from which the dermis had been removed before freezing) that had been frozen at $-17^{\circ} \mathrm{C}$ for at least $1 \mathrm{~h}$. 


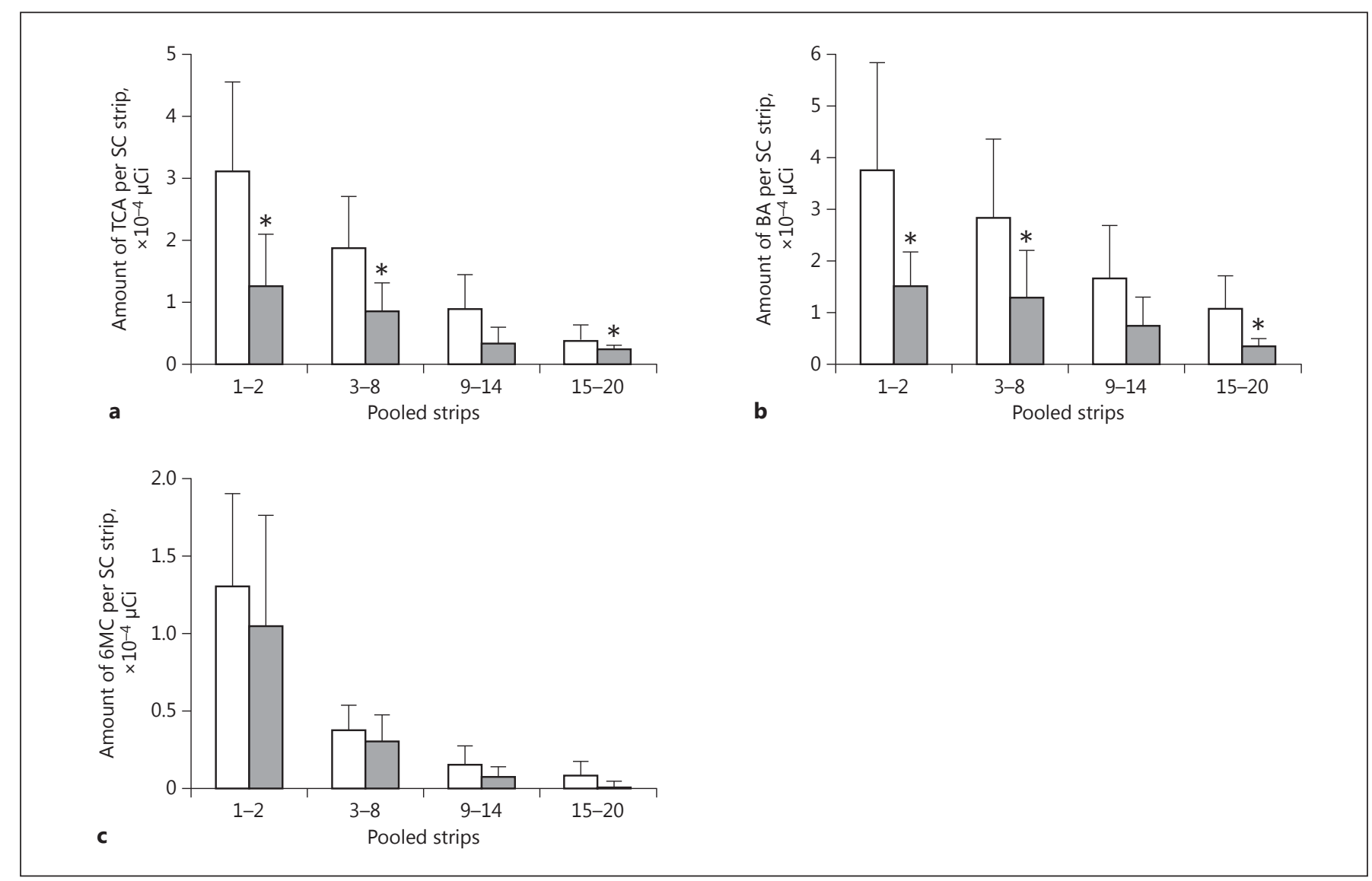

Fig. 8. Distribution of TCA (a), BA (b), and 6MC (c) across the SC $24 \mathrm{~h}$ after topical application to fresh ( $\square)$ and frozen $(\square)$ skin. Values are expressed as the amount of radioactivity $\left(\times 10^{-4} \mu \mathrm{Ci}\right)$ per strip and are a mean $\pm \mathrm{SD}$ of 12 skin discs (triplicate discs from 4 donors, with the exception of donor 3 for $6 \mathrm{MC}$ which was in duplicate). Statistical differences $(p<0.05)$ between fresh and frozen values are denoted with an asterisk.

The frozen skin samples in our studies were stored for 8-12 weeks. This allowed for leeway in testing and ensured many xenobiotic metabolizing enzymes were inactivated. A number of the 50 chemicals selected for the main studies are likely to be metabolized as they pass through the skin, and this may affect their penetration and thus the dermal delivery. However, the impact of dermal metabolism on the absorption of a metabolized chemical through skin appears to also depend on the physicochemical properties of the chemical. For example, Zalko et al. [4] showed that the penetration and distribution of the low-molecular-weight and hydrophilic compound bisphenol A in fresh and frozen skin from the same donors was the same. This is in contrast to the findings of Hewitt et al. [5], who reported that the passage of the larger-molecular-weight and lipophilic chemicals fluoxypyr esters from the SC into the epidermis required

Skin Penetration in Fresh and Frozen Human Skin cleavage of the ester to a more hydrophilic metabolite. Likewise, benzo(a)pyrene also requires metabolism to more hydrophilic metabolites before it can penetrate the epidermis and dermis $[6,7]$. With this in mind, the penetration and metabolism of some chemicals known to be metabolized by dermal metabolizing enzymes should be determined using fresh human skin.

The effect of different storage times on the penetration of chemicals has been investigated by others. Comparisons conducted using relatively shorter storage durations of 4 and 30 days (stored at $-20^{\circ} \mathrm{C}$ ) showed that there were no significant differences in the fluxes of anisole, cyclohexanone, and 1,4-dioxane between freshly excised and frozen skin [18]. Moody et al. [13] compared the penetration of 6 chemicals with varying lipophilicity $(\log P$ between -1.69 and 6.2) in dermatomed fresh skin and paired skin which had been frozen at $-19^{\circ} \mathrm{C}$ for 30 or 60 days.

Skin Pharmacol Physiol 2017;30:234-245 DOI: $10.1159 / 000475472$ 
The results depended on the parameter by which the comparison was made. There was no difference observed when the SC was excluded from the percent absorbed; however, a significant difference was noted for benzo(a)pyrene and toluene when the SC was included. Much longer storage durations of up to 1 year and 466 days were tested by Harrison et al. [19] and Bronaugh et al. [20] who reported no significant differences between the penetration of tritiated water through fresh and frozen human skin.

The dermal delivery and the distribution of TCA, BA, and $6 \mathrm{MC}$ across different skin compartments after $24 \mathrm{~h}$ were very similar in fresh and frozen skin. Small but significant differences that were observed in these studies were for $6 \mathrm{MC}$, which is likely to be related to small differences in the mass balance rather than to the skin used. Non-statistical differences for TCA and BA indicated that absorption using frozen skin was only marginally lower than the corresponding fresh skin, indicating that the barrier function was not compromised. There was also no difference in the dermal delivery between fresh and frozen skin when the SC was included in the calculation. This finding is expected since the dermal delivery of the chemicals tested here is at least 10-fold higher than the amount remaining in the SC. Thus, the amount of chemical in the SC has little impact on dermal delivery variability, which is more directly related to the amount reaching the RF, since this represents the majority of the dermal delivery (up to $76 \%$ of the applied dose).

The majority of studies report either no effect or a higher penetration of chemicals through frozen skin but there are a few studies in which the amount absorbed was lower in frozen than in fresh skin $[10,13]$. The reason for lower penetration of certain chemicals (e.g., benz(a)pyrene) in frozen skin is mainly due to inactivation of metabolizing enzymes [6,7], which is why our studies were specifically designed to compare chemicals that were not metabolized by dermal enzymes. For all 3 chemicals, the amounts recovered in the epidermis and dermis were very low and represented less than $2 \%$ of the applied dose; therefore, the statistical differences determined in these layers for BA were considered not to be biologically relevant. The amount of $6 \mathrm{MC}$ in the SC was lower in frozen than in fresh skin from donor 1 but higher in the SC of frozen than fresh skin of donor 7 (and not different in the SC from donors 5 and 6). When all donors were combined, the distribution of this chemical across the SC layers was the same. This underlines the variability between donors and that a sufficient number of donors is important for such studies. The amount of TCA and BA was lower in most layers of frozen SC than in corresponding fresh SC although this did not affect the dermal delivery of these chemicals when the SC was included in the calculation.

\section{Conclusions}

The concern that we wanted to address in these studies was that freezing the skin could damage it and thus lead to inaccurate estimations of the penetration of test chemicals - either too conservative by being much higher in frozen skin, or underestimating exposure by being much lower in frozen skin. However, although there were some statistical differences in the cumulative amount of chemical reaching the RF and the distribution across different skin layers, the penetration values for TCA, BA, and 6MC were comparable with a maximum variability factor of 2 (which could be considered not to be a biologically relevant difference).

In conclusion, the penetration of the metabolically stable chemicals TCA, BA, and 6MC was essentially unaffected by freezing the skin according to the conditions we used. This demonstrates that freezing human skin samples up to 12 weeks at $-20^{\circ} \mathrm{C}$ can be used as a standardized method to generate skin penetration data comparable to fresh skin for chemicals for which skin penetration is independent of metabolism.

\section{Acknowledgements}

We would like to thank David Sanders from Unilever Sharnbrook, UK, for all his help, including analysing the purity of 6 -methyl[2- $\left.{ }^{14} \mathrm{C}\right]$ coumarin. This work was sponsored by Cosmetics Europe.

\section{Statement of Ethics}

Abdominal human skin was ethically obtained with consent for research from donors undergoing surgery from commercial suppliers and in accordance with French laws (Banque de Tissu Lyon, France, Biopredic International, Saint-Grégoire, France, and Alphenyx, Marseille, France).

\section{Disclosure Statement}

The authors state no conflict of interest.
Jacques-Jamin et al. 


\section{References}

1 OECD, 2004a. Guidance document for the conduct of skin absorption studies. OECD series on testing and assessment (No 28), March 5, 2004.

2 OECD, 2004b. OECD guideline for the testing of chemicals. Skin absorption: in vitro method. No 428. Adopted April 13, 2004.

3 SCCS, 2010 (SCCS/1358/10). SCCS (Scientific Committee on Consumer Safety) basic criteria for the in vitro assessment of dermal absorption of cosmetic ingredients. June 22, 2010.

4 Zalko D, Jacques C, Duplan H, Bruel S, Perdu E: Viable skin efficiently absorbs and metabolizes bisphenol A. Chemosphere 2011;82: 424-430.

5 Hewitt PG, Perkins J, Hotchkiss SA: Metabolism of fluroxypyr, fluroxypyr methyl ester, and the herbicide fluroxypyr methylheptyl ester. I. During percutaneous absorption through fresh rat and human skin in vitro. Drug Metab Dispos 2000;28:748-754.

6 Jacques C, Perdu E, Duplan H, Jamin EL, Canlet C, Debrauwer L, Cravedi JP, Mavon A, Zalko D: Disposition and biotransformation of ${ }^{14} \mathrm{C}$-benzo(a)pyrene in a pig ear skin model: ex vivo and in vitro approaches. Toxicol Lett 2010;199:22-33.

7 Jacques C, Perdu E, Jamin EL, Cravedi JP, Mavon A, Duplan H, Zalko D: Effect of skin metabolism on dermal delivery of testosterone: qualitative assessment using a new short-term skin model. Skin Pharmacol Physiol 2014;27: 188.

8 Chilcott RP, Dalton CH, Emmanuel AJ, Allen CE, Bradley ST: Transepidermal water loss does not correlate with skin barrier function in vitro. J Invest Dermatol 2002;118:871-875.
9 Swarbrick J, Lee G, Brom J: Drug permeation through human skin. I. Effect of storage conditions of skin. J Invest Dermatol 1982;78: 63-66.

10 Moody RP, Martineau PA: An automated in vitro dermal absorption procedure. I. Permeation of (14)C-labelled N,N-diethyl- $m$-toluamide through human skin and effects of short-wave ultraviolet radiation on permeation. Toxicol In Vitro 1990;4:193-199.

11 Nácher A, Bossó V, Meliá M, Garrigues TM, Martin A, Díez O, Herráez M: Preliminary study with phenol red to evaluate the integrity of human epidermis in in vitro permeation experiments. http://www.sefig.org/doc/ SALAMANCA2005-pdf/BIOFARMACIA/ BIOF-005.\%20Nacher\%20y\%20col.pdf.

12 Brain KR, Walters KA, Green DM, Brain S, Loretz LJ, Sharma RK, Dressler WE: Percutaneous penetration of diethanolamine through human skin in vitro: application from cosmetic vehicles. Food Chem Toxicol 2005;43: 681-690.

13 Moody RP, Yip A, Chu I: Effect of cold storage on in vitro human skin absorption of $\operatorname{six}{ }^{14} \mathrm{C}$ radiolabeled environmental contaminants: benzo[a]pyrene, ethylene glycol, methyl parathion, naphthalene, nonyl phenol, and toluene. J Toxicol Environ Health A 2009;72:505517.

14 Hawkins GS Jr, Reifenrath WG: Development of an in vitro model for determining the fate of chemicals applied to skin. Fundam Appl Toxicol 1984;4:S133-S144.

15 Kemppainen BW, Riley RT, Pace JG, Hoerr FJ: Effects of skin storage conditions and concentration of applied dose on $\left[{ }^{3} \mathrm{H}\right] \mathrm{T}-2$ toxin penetration through excised human and monkey skin. Food Chem Toxicol 1986;24: 221-227.
16 Gerstel D, Jacques-Jamin C, Schepky A, Cubberley R, Eilstein J, Grégoire S, Hewitt N, Klaric M, Rothe H, Duplan H: Comparison of protocols for measuring cosmetic ingredient distribution in human and pig skin. Toxicol In Vitro 2016;34:153-160.

17 Fluhr JW, Feingold KR, Elias PM: Transepidermal water loss reflects permeability barrier status: validation in human and rodent in vivo and ex vivo models. Exp Dermatol 2006;15: 483-492.

18 Dennerlein K, Schneider D, Göen T, Schaller $\mathrm{KH}$, Drexler H, Korinth G: Studies on percutaneous penetration of chemicals - impact of storage conditions for excised human skin. Toxicol In Vitro 2013;27:708-713.

19 Harrison SM, Barry BW, Dugard PH: Effects of freezing on human skin permeability. J Pharm Pharmacol 1984;36:261-262.

20 Bronaugh RL, Stewart RF, Simon M: Methods for in vitro percutaneous absorption studies. VII. Use of excised human skin. J Pharm Sci 1986;75:1094-1097.

21 ACD/Labs, 2014. Percepta predictors - aqueous solubility, version 12. Toronto, Advanced Chemistry Development Inc., 2014. www. acdlabs.com

22 Willighagen EL, Denissen HM, Wehrens R, Buydens LM: On the use of ${ }^{1} \mathrm{H}$ and ${ }^{13} \mathrm{C} 1 \mathrm{D}$ NMR spectra as QSPR descriptors. J Chem Inf Model 2006;46:487-494.

23 Hansch C, Leo AJ: Database. Pomona College Medicinal Chemistry Project. Claremont, Pomona College, 1995.

24 US EPA: Estimation Programs Interface Suit$\mathrm{e}^{\mathrm{TM}}$ for Microsoft ${ }^{\circledR}$ Windows, v 4.11. Washington, United States Environmental Protection Agency, 2015.
Skin Penetration in Fresh and Frozen Human Skin
Skin Pharmacol Physiol 2017;30:234-245

DOI: $10.1159 / 000475472$ 